\section{Formación de maestros y maestras para y desde la diversidad cultural}

\section{Training of Teachers for and from Cultural Diversity}

Formação de professores

e professoras para e desde a diversidade cultural

\section{Resumen}

Este artículo presenta el resultado de una investigación sobre el contenido de la formación de maestros y maestras en Colombia que desde su Constitución Política se define como un país pluriétnico y multicultural. A través del lente que ofrece la pedagogía planetaria, como concepto y como metáfora, se realiza una lectura de las posibilidades y los retos en términos de producción y creación alternativas para pensar los diversos escenarios educativos. Entre los resultados de la investigación se destaca la importancia de pensar la formación de profesores "desde" el lente que ofrece la diversidad y no solo "para" la diversidad. Se concluye que una perspectiva como la ofrecida por esta forma de pedagogía abre un espacio para pensar formas metódicas y didácticas amplias y diversas, las cuales suponen la transformación de suelo epistemológico que sustenta las prácticas educativas reconociendo saberes locales, ancestrales y populares como marcos de acción e interpretación y no solo como objetos de estudio.

\section{Palabras clave}

Formación de maestros, educación intercultural, pedagogía planetaria.

\section{Abstract}

This article presents the result of a research on the content of the training of teachers in Colombia that from its political Constitution is defined as a multiethnic and multicultural country. Through the lens that offers the planetary pedagogy, as concept and as metaphor, is performed a reading of the possibilities and the challenges in terms of production and alternative creation for thinking the different educational scenes. Between the results of the research is highlights the importance of thinking the training of teachers "from" the lens that offers it diversity and not only "for" the diversity. A perspective as it is offered by this form of pedagogy opens a place for thinking methodical and didactic, spacious and different forms, which represent the transformation of the epistemological base that sustains the educational practices recognizing local knowledge, ancestral and popular as frames of action e interpretation and not only as objects of study.

\section{Keywords}

Teacher training, intercultural education, global pedagogy.

Fecha de recepción: junio 9 de 2016 Fecha de aprobación: junio 23 de 2016

...............................................

Artículos de Investigación

Pedagogía y Saberes No. 45 Universidad Pedagógica Nacional dor-fundador y director nacional del Grupo Historia de la Práctica Pedagógica en Colombia. Correo electrónico: jesus.echeverri@udea. edu.co

Profesora de la Facultad de Educación de la Universidad de AntioquiaUdeA, investigadora del grupo Diverser. Correo electrónico: hilda. rodriguez@udea.edu.co

* Profesor de la Facultad de Educación de la Universidad de AntioquiaUdeA, investigador del Grupo Historia de la Práctica Pedagógica en Colombia. Correo electrónico: victor.yarza@udea.edu.co

* Profesor de la Facultad de Educación de la Universidad de AntioquiaUdeA, Doctor en Historia de la educación y la pedagogía, investiga$\longrightarrow$ $\rightarrow+\infty$ 


\section{Resumo}

Esse artigo apresenta o resultado de uma pesquisa sobre o conteúdo da formação de professores e professoras na Colômbia, que desde sua Constituição Política define-se como um pais pluriétnico e multicultural. Através da lente que oferece a pedagogia planetária, como conceito e como metáfora, realiza-se una leitura das possibilidades e os desafios em termos de produção e criação alternativas para pensar os diversos cenários educacionais. Entre os resultados da investigação destaca-se a importância de pensar a formação de professores "desde" a lente que oferece a diversidade e não só "para" a diversidade. Conclui-se que uma perspectiva como a oferecida por essa forma de pedagogia abre um espaço para pensar formas metódicas e didáticas amplias y diversas, as quais supõem a transformação da base epistemológico que sustenta as práticas educacionais reconhecendo saberes locais, ancestrais e populares como marcos de ação e interpretação e não só como objetos de estudo.

\section{Palavras-chave}

Formação de professores, educação intercultural, pedagogia planetária.

\section{Introducción}

$\mathrm{L}$ a declaración constitucional de que somos un país pluriétnico y multicultural no es suficiente para lograr que el reconocimiento de la diversidad sea una propuesta política; y mucho menos para indicar que se ha tomado los espacios institucionales. La educación no escapa a ello. Mucho se ha hablado de la educación para o desde la diversidad cultural, sin que se pueda indicar cómo se integra este componente a las actividades escolares, sin que pasemos del reconocimiento de costumbres de las comunidades afro e indígenas; o sin que celebremos el Día de las Identidades (antes llamado Día de la Raza), el Día de la Afrocolombianidad.

El auge de las reformas (constitucionales) en que la diversidad da paso al reconocimiento de derechos jurídicos a "las minorías", se articula a nuevas prácticas (políticas y educativas) para la inclusión. Y la formación de maestros es una de estas prácticas; por ello, es frecuente encontrar cursos, seminarios, congresos y demás espacios de formación que promueven la presencia, la comprensión y la puesta en práctica de la diversidad. Sin embargo, este nivel discursivo no alcanza a transformar las prácticas escolares o los planes de formación de docentes en las facultades de educación.

Pese a que, desde el discurso, la diversidad se reconoce como algo positivo y necesario, como una potencia, en la práctica nos encontramos con denominaciones que la ponen del lado de lo problemático. A veces se la señala como el origen de algunos males escolares, como el fracaso, las dificultades en la convivencia o las limitaciones para el aprendizaje. Esta tensión entre el discurso y la práctica nos lleva a preguntarnos si la formación de maestros y maestras en diversidad cultural se convierte en una opción académica y pedagógica.

\section{Preposiciones}

Para dar respuesta a nuestro interrogante, usamos dos preposiciones: para y desde. En el Diccionario de la lengua española de la Real Academia de la Lengua se presentan los siguientes significados de estas preposiciones:

\section{Para}

Denota el fin o término a que se encamina una acción.

Denota sentido de un movimiento.

Úsase para determinar el uso que conviene o puede darse a algo.

Úsase como partícula adversativa, significando el estado en que se halla actualmente algo, contraponiéndolo a lo que se quiere aplicar o se dice de ello.

Denota la relación de una cosa con otra, o lo que es propio o le toca respecto de sí misma.

\section{Desde}

Denota el punto, en tiempo o lugar, de que procede, se origina o ha de empezar a contarse una cosa, un hecho o una distancia.

Úsase para introducir la perspectiva, el enfoque, el aspecto o la opinión que se expresan.

Con los pronombres personales mí, sí, etc., y con algunos verbos, denota la particularidad de la persona, o que la acción de lo expresado por el verbo es interior, secreta y no se comunica a otro. 
Para nosotros, el uso de estas dos preposiciones debe darse de manera simultánea, para describir y explicar la formación de maestros y maestras; para evitar que se quede en una orilla u otra. A continuación, explicamos nuestra perspectiva.

\section{Formación para la diversidad}

Está encaminada a:

- la comprensión de los conceptos implicados en la diversidad cultural, la claridad de los supuestos (éticos, políticos, sociales), a la articulación de las ideas al respecto.

- la ética, el cuidado, el respeto y la justicia social que nos permitan reconocer a los demás como fuentes de saber.

- comprender la diversidad epistémica, de manera que nos permita superar los etnocentrismos, la exotización de los demás.

La formación para el saber, el concepto y la comprensión histórica de la interculturalidad; para el reconocimiento de las condiciones jurídicas y políticas de la diversidad. Para, como fin de la formación de maestros y maestras, significa un punto de partida ilustrado sobre la diversidad, la interculturalidad, y convertir ello en norma de acción para la práctica.

\section{Formación desde la diversidad}

- Nos habla del punto de partida de la acción pedagógica: la diversidad, el encuentro, el diálogo; la búsqueda de orientaciones para construir espacios múltiples donde albergar la diversidad.

- El enfoque o perspectiva, el sello de la formación, la marca o impronta de las reflexiones y la acción.

Usar la preposición desde, nos permite enfatizar que la formación también debe abordarse desde la diversidad, en el campo metódico y didáctico, requiere amplitud, dispersión, necesita enfatizar en diversas formas de compromiso, acción, actividad. Que las aproximaciones, también, tienen que ver con lo epistemológico; esto es, con el reconocimiento de los saberes (locales, ancestrales, populares) como marcos de acción e interpretación, y no solamente como objetos por tratar.

Así, podemos presentar dos ámbitos de formación de docentes: el teórico, que indaga por cuestiones relativas a las nociones, las posturas políticas y sociales sobre los grupos, indagaciones epistémicas sobre el saber (ancestral, popular, situado), cuestiones sobre las relaciones entre lenguaje, pensamiento, cultura. De otro lado, se encuentran los asuntos prácticos, referentes a cómo enseñar; esto es, asuntos referidos a la práctica educativa; sin que se aborden los temas relativos a qué enseñar, ¿cuáles son los contenidos de la diversidad cultural? que a veces se resuelve con un proyecto transversal (el caso de la Cátedra de Estudios Afrocolombianos), en los que se perpetúan visiones exotizantes de los demás.

Estos ámbitos no están separados; los une el uso de las preposiciones; para lograr una formación desde y para la diversidad; una formación que integre el cómo y el qué; que articule la idea y la acción. Con esta articulación, podemos hacer actuales el verso de Machado: "Caminante, no hay camino, / se hace camino al andar."; al movernos, al cambiar de postura, al modificar el punto de vista. Además, los versos indican que el inicio de esta formación se encuentra ubicado en la labor personal que deben hacer los y las docentes para reconocer sus propias concepciones y orientaciones, no como hablantes de una realidad sino como actuantes de una práctica.

La articulación entre desde y para nos indica que no se trata solamente del dominio teórico o la calidad de la práctica. También está relacionado con la imagen de mundo que pueden compartir los y las docentes con sus estudiantes; significa, además, asumir el compromiso político del oficio de enseñar: aportar al cambio social. Formar desde y para la diversidad, de manera concreta, implica:

- Revisar las metas de la educación, aquellas que propone la Ley General de Educación.

- Concebir la diversidad como lo propio del género humano, no como una condición o característica de ciertos grupos.

- Promover valores como la empatía, la cooperación y el trabajo conjunto.

- Reflexionar sobe las propias prácticas para develar los gestos inadecuados, los sesgos limitantes de una visión, las metáforas explicativas que reducen el mundo.

- Complementar las formas de aprender y enseñar, de construir y transmitir conocimientos.

\section{Educación y diversidad cultural}

Desde los eleatas hasta la fecha el pensamiento dialéctico ha tenido tiempo de sobra para darnos sus frutos. Los estamos comiendo, son deliciosos, hierven de radiactividad. Y al final del banquete, ¿por qué estamos tan tristes?...

Cortázar 
La educación desde y para la diversidad cultural es un núcleo de reflexiones en el cual se unen definiciones y conceptos como cultura, identidad, formación, saberes autorizados, en una aproximación multicultural al tema, en tanto estado, permanencia, confluencia sin interacción, coexistencia pacífica y aceptación a-crítica de los supuestos. Lo que en palabras de Aguado (2003) está basado en un modelo compensatorio:

\begin{abstract}
Los modelos educativos compensatorios intentan hacer frente a los modelos educativos selectivos, definidos por valores que no reconocen la diversidad humana o que la definen en términos jerárquicos en los que unas formas de pensar, hacer o ser son superiores a otras. Estos modelos se operativizan en programas que corresponden a proyectos de centro más institucionales y articulados cuyos objetivos son promocionadores, al concebir el progreso educativo como ascenso de un nivel superior a otro superior. (p. 10)
\end{abstract}

La tarea de la diversidad es ofrecer otros marcos de explicación y descripción de las realidades escolares, de modo tal que se trastoquen las políticas de identificación, reconocimiento de producción de saberes y enseñanza. Ello nos aproxima a una versión intercultural de la educación, desde y para la diversidad, lo que nos alienta en la construcción de perspectivas, miradas y formas de producción de conocimiento. Como advierten Rojas y Castillo (2007), la interculturalidad

\section{... no es solo una forma de establecer relaciones entre saberes distintos, sino un proyecto político que cuestiona los lugares de poder desde los cuales se producen las representaciones mismas acerca de lo que es, y lo que no, reconocido como conoci- miento. (p. 21)}

Y en el caso de la formación de maestros y maestras, el tránsito de lo multicultural a lo intercultural se comprende en lo que Juan García denomina casa dentro/casa fuera, para lo cual es necesario hablar de otras prácticas, identificar otras educaciones, hacer surgir otros saberes, reconocer lógicas, epistemes, cosmogonías.

\begin{abstract}
... Casa adentro, en este sentido significa asumir una agencialidad y posicionamiento hacia la producción de conocimientos, a cambiar el lente eurocéntrico y ver desde las historias vividas, promoviendo así una fortaleza colectiva. Y es esta fortaleza colectiva la que permite negociar de otra manera el trabajo de casa afuera. (García, citado en Walsh, 2007, pp. 32-33).
\end{abstract}

Queremos precisar aquí que no se trata de la formación de maestros y maestras para la etnoeducación; esto es, como señalan Castillo y Caicedo (2010), una educación para los otros. En nuestro caso, es una propuesta de formación para una educación intercul- tural en los diversos contextos escolares: urbanos, rurales, centrales, periféricos, comunes, especializados, diferenciales y para personas en situación de discapacidad, y con capacidades y talentos excepcionales. Pues concebimos que la perspectiva intercultural no es (solamente) étnica; sino que se articula a los diversos escenarios en los cuales los seres humanos interactuamos, nos relacionamos, convivimos y vivimos. Así, la formación intercultural procura el descentramiento, la mirada amplia, la ruptura de la episteme clásica. En esa lógica, Medina señala:

La educación intercultural en nuestro continente puede ser repensada como proyecto político y pedagógico, implica la construcción activa de espacios de formación de los sujetos sociales que hacen eco del reclamo de los derechos históricos sobre la lengua, la memoria y el territorio. (2009, p. 13)

Ello nos indica que la perspectiva intercultural de la educación pone en tensión maneras de concebir la realidad, espacios de socialización, las políticas de conocimiento (Apple, 1994) que ubica en el horizonte de la formación acciones y concepciones de comunidades, pueblos, organizaciones; para inventar otras maneras de ser docente, poner en juego otros saberes, lenguajes, prácticas, ideales formativos, relacionamientos, cosmovisiones.

Nuestra propuesta de una formación de maestros desde y para la diversidad tiene como base una aproximación a la noción de saber como "aquello de lo que se puede hablar en una práctica discursiva, específicamente como el campo que está constituido por diferentes objetos que adquirirán o no un estatuto científico" (Foucault, 1997, p. 306). En otras palabras, no existe saber sin una práctica discursiva; lo que en el marco de este artículo se comprende como que no es posible integrar saberes otros a la formación de maestros y maestras, si no se acompaña de una práctica entendida como

... la racionalidad o regularidad que organiza lo
que los hombres hacen - sistema de acción en la
medida en que están habitados por el pensamiento,
(objeto de reflexión y análisis) - que tienen un
carácter sistemático (saber, poder, ética) y general
(recurrente), y que por eso constituye una expe-
riencia "o un pensamiento". (Castro, 2004, p. 427)

Es decir, la práctica como red, conexión, como lupa examinadora de las condiciones naturalizadas de circulación e inscripción de saberes, para abrir fisuras, la práctica como espacio singular de producción y sentido, tal como señala Haraway (1995): "No buscamos la parcialidad porque sí, sino por las conexiones y aperturas inesperadas que los conocimientos situados hacen posibles. La única manera de encontrar una visión amplia es estar en algún sitio en particular". (p. 339) 


\section{Una superficie de análisis}

En estas coordenadas generales, hemos constatado la hegemonía de la monoculturalidad y multiculturalidad en la formación de maestros en Colombia (1994-2015), expresada tanto en su normativa nacional, regional, local e incluso institucional, como en diversos documentos del sistema de formación docente, lineamientos e incluso debates de académicos, intelectuales, formadores de maestros, y los mismos maestros en formación, entre otros. La relación con el contexto internacional y la globalización aparece tecnificada, y utilizada como argumento de obligatoriedad. No trasciende en su conexión con la interculturalidad, la planetariedad, o el cuidado. Una vez más, se evidencia la urgencia de interculturalizar la formación docente en nuestro país.

En una periodización todavía provisional, se identificó que entre 1994 y 2002 la diversidad en la formación de maestros aparecía mínimamente desde las siguientes aristas:

- Desde la especificidad o énfasis poblacional en las licenciaturas, es decir, tanto en sus denominaciones como en sus sujetos y objetos de formación: Licenciatura en Etnoeducación, en Educación Especial, Educación Campesina (cfr. Ley 115 de 1994).

- Desde los fines, principios y objetivos de la normativa general y diferencial ( $c f r$. Ley 115 de 1994, o Decreto 804 de 1995).

- Mediante los núcleos del saber pedagógico, propuestos desde el corazón del Movimiento Pedagógico Nacional, específicamente: educabilidad, y tendencias y contextos ( $c f r$. Decreto 3012 de 1996 o Decreto 272 de 1998). Acá la diversidad es del otro, sin trastocar las condiciones de la relación o la mismidad en sí. A lo largo de la periodización aparece una multiculturalidad centelleante: a veces intensa, otras difusa en el horizonte, siempre multicultural. Acá las competencias tienen fuerte énfasis disciplinar, pedagógico y didáctico de ciencias de la educación.

Al mismo tiempo, la interculturalidad y la etnoeducación (y sus variaciones posteriores como la educación propia, la afroetnoeducación, entre otras), son subalternizadas, invisibilizadas y marginadas en las discusiones normativas/disciplinares en la pedagogía para la formación de maestros, mientras que adquiere una mayor consistencia, solidez y vitalidad en los movimientos sociales indígenas y afrodescendientes (cfr. Castillo y, 2015).
En una segunda periodización, aproximadamente entre 2003 y 2013, el panorama es más difuso desde el punto de vista discursivo, por cuanto coexisten modelos o perspectivas antagónicas en aparente articulación o convivencia. Mientras, el gerencialismo escolar del Estado evaluador promueve la estandarización, homogeneización y normalización/regulación de los procesos formativos, sus saberes, prácticas, sujetos e instituciones; permanentemente toma fuerza y se edifica en los imperativos modernos de inclusión, igualdad o equidad, desde una irrefutable celebración de la diversidad. Un antagonismo que agudiza la presencia de la mono-multiculturalidad, y del asimilacionismo/integracionismo dominante en los modelos de escolarización de las diferencias culturales en nuestras sociedades.

Recientemente, los lineamientos del sistema de formación de maestros (2014) y el decreto de ajuste radical a las instituciones de formación de maestros (Decreto 2450 de 2015) evidencian esa contradicción constitutiva y paradojal que signa nuestros días: mientras que en 2014 aparecen la interculturalidad y la diversidad cultural como un discurso estructurante y complementario de la formación inicial (aunque no se vea reflejado contundentemente en las competencias: enseñar, formar y evaluar), el decreto instrumentaliza y reduce al mínimo la expresión multicultural.

Por tanto, se considera absolutamente necesario y urgente continuar desestabilizando e interculturalizando la formación inicial de educadores, desde perspectivas subalternas, insumisas, libertarias, críticas, holísticas, interculturales, todas estas y más, pero a una nueva escala: la planetaria. El desafío freireano de humanizar la educación trasciende a la humanización de la globalización, o mejor dicho la planetariedad.

\section{Pedagogía planetaria y formación de maestros y maestras}

La pedagogía planetaria es una metáfora que nos sirve para aludir a la formación de maestros y maestras como prácticas dispersas, difusas y contextuales; que leen el presente y reconocen el pasado; que identifican, en el mapa de las interacciones, aquellos saberes que han sido excluidos de los ámbitos de formación, para hacerlos notar, subrayarlos, ponerlos de presente y develar la manera como actúan, los espacios que copan, los lenguajes que inauguran y las acciones que promueven. Con ello se dibuja un nuevo mapa de la formación, que da cuenta de otras dimensiones y espacios. 
En las facultades de educación, la pedagogía planetaria no solo rescata, visibiliza, desata los saberes subalternos, minorizados y subyugados. También tiene como tarea formular preguntas a las pretensiones reduccionistas de la formación y el ejercicio del oficio ${ }^{1}$, desconfigurar la perspectiva técnicaeficientista que ha relevado el sentido de la artesanía en la formación.

Para ello, la pedagogía planetaria recurre a conceptos, teorías y experiencias (la noción de campo nos resulta útil), para (de)mostrar las dimensiones del sujeto maestro, las relaciones con el saber y el encuentro con los demás sujetos de la práctica pedagógica. La pedagogía planetaria es una brújula para cruzar las fronteras de que nos habla Giroux (1997):

Ser un cruzador de fronteras significa que uno tiene que descubrir de nuevo tradiciones, no dentro del discurso de sumisión, reverencia y repetición sino, como escribe Ernesto Laclau, "como transformación y crítica. [Esto es,]... uno debe construir su propio discurso como diferencia respecto a esa tradición, y eso supone al mismo tiempo continuidades y discontinuidades". (p. 222)

La pedagogía planetaria es herencia y legado; es recepción de tradiciones y construcción de alternativas en diálogo y revisión; es campo de validación y campo de referencia, espacio de producción y creación. La pedagogía planetaria es práctica y acción.

La pedagogía planetaria es, también, síntesis o conjugación; y en tanto "concepto utillaje" (Rodríguez y Yarza, 2016) da cuenta de la apropiación de prácticas, concepciones, ideas, postulados de tradiciones que se encuentran arraigadas: como el caso de la etnoeducación o de la educación bilingüe intercultural. De ellas retoma su orientación política, las diferencias de perspectiva (encuentro, resistencia, aculturación) y enfoque (para las minorías, para los otros). La pedagogía planetaria también busca poner en cuestión lógicas hegemónicas (Rojas y Castillo, 2005) en la formación de quienes fungen como educadores, y recuperar diversas figuras de interacción, saber y autoridad. Esto ya fue posible en la experiencia que el Consejo Regional Indígena del Cauca CRIC (2004) relata sobre los procesos de profesionalización de sus maestros:

1 Para comprender la expresión oficio de maestro, nos valemos de las palabras de Saldarriaga (2003): "Prefiero hablar de 'oficio de maestro', porque defiendo el peso histórico y cultural del término maestro, que define a la persona dedicada al oficio de enseñar, frente a otros términos más recientes que no añaden nada esencial a esa designación. Aunque debe precisarse de inmediato que términos como profesor, docente, educador, pedagogo, califican ciertos matices en la jerarquía social y académica para distinguir diversas especies dentro del mismo género".
La formación pedagógica no era exclusiva de los docentes, sino que se extendía a la dirigencia local, a la comunidad, al equipo coordinador y a la misma organización regional. A través de este proceso se revitalizó el papel del maestro frente a la comunidad, porque dejo de ser el único portador de conocimiento, para convertirse en un coordinador y articulador de los distintos saberes. (CRIC, 2004, p. 56)

Así, la pedagogía planetaria retoma la formación como eje de acción de sujetos que, en diversas prácticas, se llevan a cabo a través de sabedoras, sabias, lideresas. Esto tiene, al menos, dos efectos notables. De un lado, se descentra la educación de la figura del maestro o maestra, y se recupera la visión de la comunidad educadora; de otro, se desescolariza; esto es, la escuela no es el centro de las acciones o actividades, sino un espacio más de formación al lado de los rituales, los encuentros, las prácticas de palabra y pensamiento. A este respecto Tenorio (2011) se pregunta si la escolaridad generalizada es inclusión social o pérdida de la identidad cultural. Las palabras de García (2007, p. 4) las podemos tomar como una respuesta:

Ante la invasión magisterial, la educación tradicional indígena retrocede y retrocede. Se "encoge", pues los más jóvenes han de pasar muchas horas, cada día, entre las paredes de la escuela, sustraídos a la interacción comunitaria. Los niños estarán menos tiempo que nunca al lado de sus mayores, al lado de esos ciudadanos que encarnan los valores "locales", étnicos; y más tiempo que nunca bajo el poder de estos extraños que difunden los principios "nacionales", occidentales [...] [los profesores] arrancan a los niños de la vida real, de la relación cotidiana con el medio, del trabajo cooperativo, de las actividades grupales, de los tiempos y lugares en los que la comunidad se recogía para fortalecerse y enjoyarse.

La pedagogía planetaria no proviene de movimientos sociales o comunitarios; tampoco es producto de luchas intelectuales o políticas por el reconocimiento (desde las políticas de la diferencia o desde la aceptación de la diversidad); no pretende convertirse en política educativa; no insiste en la idea de que la interculturalidad o la etnoeducación aluden a las obligaciones o demandas de los sujetos con marcas étnicas (indígenas, afro, palenqueros, raizales o rom) de relacionarse con esos "otros" que no portan una marca étnica y son "mayoría" poblacional en el territorio nacional. Ello la diferencia de otras iniciativas, como la etnoeducación, la educación indígena, los modelos intercultural bilingüe o bilingüe intercultural. Nuestra pretensión es que se instituya como base de la formación de maestros y maestras, que abra escenarios para el cruce de saberes y la intersección de sujetos participantes en las prácticas educativas, 
se torne en perspectiva para la educación. En otras palabras, debe ser un proyecto (político, social y cultural) para maestros y maestras; presente en su formación inicial y tornarse voluntad, disposición, capacidad y cualidad de los y las enseñantes.

En el marco de un campo conceptual y narrativo de la pedagogía (Echeverri, 2009), la pedagogía planetaria retoma lo que propone Bourdieu (2000) sobre el habitus: "el conocimiento y el reconocimiento de las leyes inmanentes de los objetos en juego" (p. 112). Para el caso de la pedagogía planetaria, se trata de las relaciones, tensiones, disputas, rupturas, continuidades, articulaciones, desplazamientos, solapamientos entre los conceptos y prácticas en la formación de maestros y maestras. En esa medida, se trata de reconfigurar las posiciones (de enunciación, de acción, de incidencia política), los sujetos que intervienen y participan, los saberes que entran en juego, por eso aludimos a la pedagogía planetaria como metáfora "que posibilita visibilizar los saberes sometidos, subalternizados, minorizados o invisibilizados" (Rodríguez y Yarza, 2016). De este modo, y de acuerdo con Bourdieu (1997)

... el habitus cumple una función que, en otra filosofía se confía a la conciencia trascendente: es un cuerpo socializado, un cuerpo estructurado, un cuerpo que se ha incorporado a las estructuras inmanentes de un mundo o de un sector particular de este mundo, de un campo, y que estructura la percepción de este mundo y también la acción en este mundo. (p. 146)

Con lo que nos será posible dar cuenta de (otros) movimientos, iniciativas, prácticas, propuestas sobre la formación de maestros y maestras que supera dicotomías entre oficios, perspectivas, espacios de producción y reproducción de saberes. El habitus alude

al mismo tiempo a un "oficio", un capital de técnicas, de referencias, un conjunto de "creencias", propiedades que se deben a la historia (nacional e internacional) de la disciplina, a su posición (intermedia) en la jerarquía de las disciplinas, y que son a la vez condición del funcionamiento del campo y el producto de ese funcionamiento (pero no integralmente: un campo puede contentarse con acoger y consagrar un tipo determinado de habitus que ya están más o menos completamente constituidos). (Bourdieu, 2000, p. 113)

La pedagogía planetaria, en cuanto campo, destaca las tensiones que se presentan en su doble realización: como proyecto político y cultural y como dispositivo/ estrategia de transformación de la sociedad desde la escuela, como espacio propicio para la creación de una conciencia/identidad nacional.

\section{Referencias bibliográficas}

Aguado, T. (2003). Pedagogía intercultural. Madrid: McGraw-Hill.

Apple, M. (1994). Educación y poder. Madrid: Paidós.

Bourdieu, P. (1997). Razones prácticas, sobre la teoría de la acción. Barcelona: Anagrama.

Bourdieu, P. (2000). Cuestiones de sociología. Madrid: Istmo.

Castillo, E. y Caicedo, J. A. (2010). Las luchas por otras educaciones en el bicentenario: de la Iglesia-docente a las educaciones étnicas, Nómadas, 33, 109-127.

Castillo, E. y Guido, S. (2015). La interculturalidad: ¿principio o fin de la utopía? Revista Colombiana de Educación, 69. 17-44.

Castro, E. (2004). El vocabulario de Michel Foucault. Un recorrido alfabético por sus temas, conceptos y autores. España: Universidad Nacional de Quilmes.

Consejo Regional Indígena del Cauca (CRIC). (2004). ¿Qué pasaría si la escuela...? 30 años de construcción de una educación propia. Popayán: El Fuego Azul.

Cortázar, J. (2013). Rayuela [edición conmemorativa 50 años]. España: Alfaguara.

Echeverri, A. (2009). Un campo conceptual de la pedagogía: una contribución. Cali: Universidad del Valle.

Foucault, M. (1997). La arqueología del saber. México: Siglo XXI.

Giroux, H. (1997). Cruzando límites. Trabajadores culturales, y políticas educativas. Barcelona: Paidós.

Haraway, D. (1995). Ciencia, cyborgs y mujeres. La reinvención de la naturaleza. Madrid: Cátedra.

Medina, P. (2009). Repensar la educación intercultural en nuestras américas. Decisio, 24, 3. Número especial.

Rodríguez, H. y Yarza, A. (2016). Formación de educadores desde una pedagogía planetaria. Magisterio, 78.

Rojas, A. y Castillo, E. (2007). Multiculturalismo y políticas educativas en Colombia, interculturalizar la educación? Educación y Pedagogía, 19(48), 11-24.

Rojas, A. y Castillo, E. (2005). Educar a los otros. Estado, políticas educativas y diferencia cultural en Colombia. Popayán: Editorial Universidad del Cauca.

Tenorio, M. (2011). Escolaridad generalizada: ¿inclusión social o pérdida de la identidad cultural? Revista de Estudios Sociales, 40, 57-71.

Walsh, C. (2007). Interculturalidad, colonialidad y educación. Educación y Pedagogía, 19(48), 25-35. 
